\title{
AVALIAÇÃO DO ESTADO NUTRICIONAL DE MULHERES IDOSAS RESIDENTES DA REGIÃO NORDESTE DO BRASIL ATRAVÉS DE DADOS DO SISVAN-WEB
}

\author{
ASSESSMENT OF THE NUTRITIONAL STATUS OF ELDERLY WOMEN LIVING IN THE \\ NORTHEAST OF BRAZIL USING DATA FROM SISVAN-WEB
}

\author{
Laís Romeica Soares da Silva ${ }^{1}$ \\ Angélica de Kássia Barbosa Flôr Monteiro² \\ Nathalia Karolyne de Andrade Silva ${ }^{3}$ \\ Maria Eduarda Francisca Xavier ${ }^{4}$
}

RESUMO: OBJETIVO: O presente estudo tem por objetivo avaliar o estado nutricional de mulheres idosas que residem na Região Nordeste do Brasil, durante o período de I ano e 2 meses. MÉTODO: Refere-se a um estudo transversal, com características descritivas tendo como linha de pesquisa idosos com idade de 60 anos ou mais, do sexo feminino. Dados obtidos a partir da plataforma SISVAN-WEB foram incluídas mulheres idosas residentes da Região Nordeste do Brasil, sem restrição de cor, povo/comunidade e área de cobertura. RESULTADOS: Os relatórios foram obtidos através do SISVAN-WEB, durante I4 meses de avaliação com Amostra total de 5.925 idosas dos 9 estados do Nordeste do Brasil. Observa-se que em janeiro de 2019 houve uma prevalência de baixo peso $(<20 \%)$, eutrofia $(<30 \%)$ e sobrepeso $(>50 \%)$. Outro mês que pode ser destacado é abril onde encontrou-se um aumento de idosas com diagnóstico de eutrofia e baixo peso, porém no mês posterior (maio) observou-se um aumento de sobrepeso. No mês de outubro de 2019 houve uma frequência de $56 \%$ de sobrepeso (sendo a maior do período de avaliação) e no mês de abril do mesmo ano 42\% (sendo o menor índice). CONCLUSÃO: O presente estudo sugere que o estado nutricional de mulheres idosas residentes da Região Nordeste encontradas no SISVAN-WEB pode não evidenciar um resultado tão fidedigno do diagnostico nutricional, pois diversos parâmetros corroboram para melhor avaliação do idoso, não apenas o IMC e avaliação do consumo alimentar, que são os métodos que a plataforma utiliza.

Palavras-chave: Estado nutricional. Pessoas Idosas. Avaliação nutricional.

ABSTRACT: OBJECTIVE: The present study aims to assess the nutritional status of elderly women living in the Northeast of Brazil, during the period of $\mathrm{I}$ year and 2 months. METHOD: Refers to a cross-sectional study, with descriptive characteristics having as a research line elderly women aged 6o years or over, female. Data obtained from the SISVAN-WEB platform included elderly women living in the Northeast of Brazil, without color restrictions, people / community and coverage area. RESULTS: The reports were obtained through SISVAN-WEB, during I4 months of evaluation with a total sample of 5,925 elderly women from 9 states in the Northeast of Brazil. It is observed that in January 2019 there was a prevalence of underweight $(<20 \%)$, normal weight $(<30 \%)$ and overweight (>

\footnotetext{
Faculdade de Comunicação, Tecnologia e Turismo de Olinda, graduada Nutrição. E-mail: laisromeica@hotmail.com

${ }^{2}$ Faculdade de Comunicação, Tecnologia e Turismo de Olinda, Bacharelado Nutrição.

${ }^{3}$ Nutricionista, coorientadora, especialista em saúde do Idoso pelo IMIP.

${ }^{4}$ Faculdade de Comunicação, Tecnologia e Turismo de Olinda, graduada Nutrição.
} 
50\%). Another month that can be highlighted is April, where there was an increase in elderly women diagnosed with eutrophy and low weight, but in the following month (May) an increase in overweight was observed. In October 2019 there was a frequency of $56 \%$ of overweight (the highest in the evaluation period) and in April of the same year 42\% (the lowest index). CONCLUSION: The present study suggests that the nutritional status of elderly women living in the Northeast Region found in SISVAN-WEB may not show such a reliable result of the nutritional diagnosis, since several parameters corroborate for a better evaluation of the elderly, not only the BMI and evaluation of the food consumption, which are the methods that the platform uses.

Keywords: Nutritional status. Old people. Nutritional assessment.

\section{INTRODUÇÃO}

De acordo com a Organização Mundial da Saúde (OMS), a idade de classificação do idoso é de 60 anos ou mais ${ }^{1}$. O Brasil tem mais de 28 milhões de pessoas nessa faixa etária, número que representa $13 \%$ da população do país. Enquanto na região nordeste temos 4.767 .906 idosos que representa 8,4\%, segundo a Projeção da População, divulgada em 2018 pelo IBGE$^{2}$, sendo as mulheres a maioria expressiva nesse grupo 3 .

Com o processo de envelhecimento, ocorre a tendência fisiológica de ganho de peso pelo acúmulo de tecido adiposo, que pode ser identificada nas avaliações física e nutricional de acompanhamento do indivíduo ${ }^{4}$. $\mathrm{O}$ excesso de peso é um problema de saúde pública e tem forte associação com o risco e a prevalência de doenças crônicas não transmissíveis. Os idosos tem maior probabilidade de serem acometidos por suas alterações metabólicas ${ }^{5}$.

O Índice de Massa Corporal (IMC) é o método antropométrico mais utilizado na avaliação nutricional, por ser uma medida de fácil e rápida execução, além de baixo custo, e esse pode identificar excesso de peso ${ }^{6}$. Diferentes fatores favorecem o ganho de peso na terceira idade, entre eles destaca-se o excesso e dequilíbrios no consumo de nutrientes ingeridos e sedentarismo ${ }^{7}$.

A partir da avaliação nutricional, pode-se realizar planejamentos e intervenções adequadas ao tratamento, recuperação e promoção da saúde dos idosos ${ }^{8,9}$. A avaliação nutricional para idosos oferece um perfil diferenciado e personificado em comparação aos demais grupos populacionais ${ }^{\mathrm{io}}$.

No Brasil, o estado nutricional é acompanhado por amostras de usuários, com aferição de medidas antropométricas (peso, altura, circunferências) e entrevista de consumo alimentar, com 
dados tabulados no SISVAN (Sistema de Vigilância Alimentar e Nutricional), ferramenta desenvolvida pelo DATASUS ${ }^{\text {II }}$.

O presente estudo tem por objetivo avaliar o estado nutricional de mulheres idosas que residem na Região Nordeste do Brasil, durante o período de I ano e 2 meses.

\section{MÉTODO}

Refere-se a um estudo transversal, com características descritivas tendo como linha de pesquisa idosos com idade de 6o anos ou mais, do sexo feminino. Os dados foram obtidos a partir da plataforma SISVAN-WEB, após incluir na amostra mulheres idosas residentes da Região Nordeste do Brasil, sem restrição de cor, povo, comunidade e/ou área de cobertura.

Para o presente estudo foram excluídos idosos do sexo masculino e pessoas idosas do sexo feminino residentes de outras regiões (Sul, Sudeste, Centro-Oeste e Norte do Brasil).

Foi avaliado o estado nutricional através do IMC, com o ponto de corte pela classificação Lipschitz (1994) com dois ponto de corte $\left(22,0 \mathrm{Kg} / \mathrm{m}^{2}\right.$ e $\left.27,0 \mathrm{Kg} / \mathrm{m}^{2}\right)$, tendo resultados baixo peso

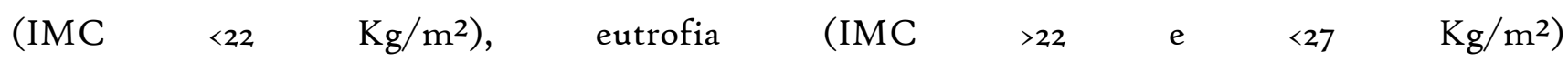
e

sobrepeso $\left(\mathrm{IMC}>27 \mathrm{Kg} / \mathrm{m}^{2}\right)$, durante o período de janeiro/2019 a fevereiro/2020. Os dados foram digitados no programa Excel para Windows ${ }^{\circledR}$ para as análises de frequência.

\section{RESULTADOS}

Os relatórios obtidos através do SISVAN-WEB durante I4 meses apresentaram 5.925 idosas como amostra total dos 9 estados do Nordeste do Brasil.

Houve um crescimento significativo de idosas com sobrepeso, comparado ao baixo peso e eutrofia do mesmo período, como pode ser observado no gráfico da Figura I.

Observa-se que em janeiro de 2019 houve índices correspondentes ao baixo peso (<20\%), eutrofia (<30\%) e sobrepeso ( $>50 \%)$.

Outro mês que pode ser destacado é abril onde há um aumento de idosas com diagnóstico nutricional de eutrofia e baixo peso, porém no mês seguinte (maio) esses percentuais foram $35 \%$ e I2\%, respectivamente, e com sobrepeso ainda predominante.

Em outubro de 2019, houve um índice de 56\% de sobrepeso (sendo o maior no período de avaliação), e no mês de abril do mesmo ano, indicou 42\% (sendo o menor índice). 
Comparando os meses de fevereiro do ano de 2019 e 2020, podemos observar que os dados são bem proporcionais, mas indicam uma leve redução do sobrepeso.

Figura I - Gráfico do estado nutricional da Região Nordeste

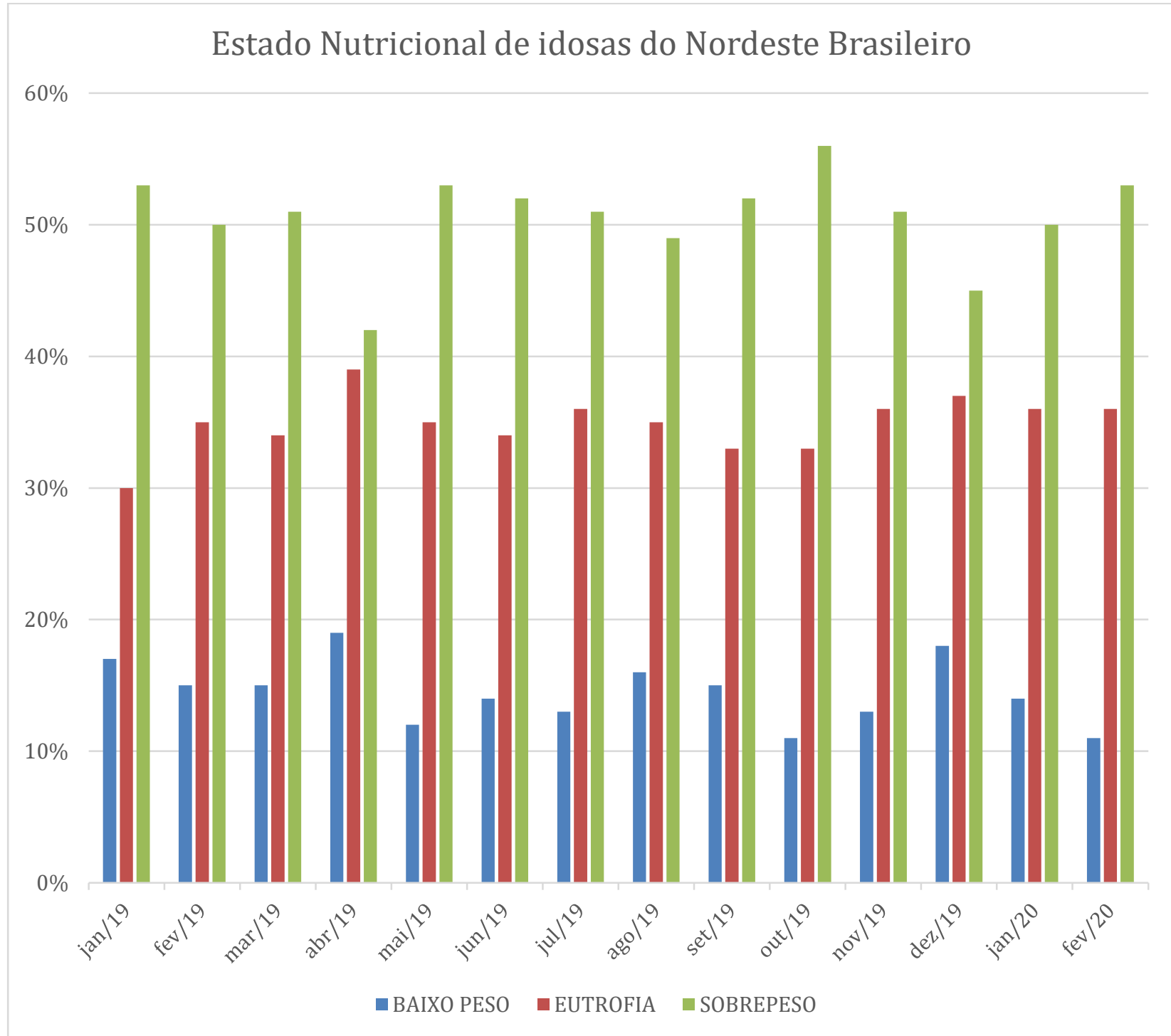

Quando isolado, o sobrepeso oscila entre 40\% e 60\%, superando o percentual de $50 \%$ principalmente nos meses de janeiro, maio, outubro de 2019 e fevereiro de 2020, como pode ser observado no gráfico da figura 2. 
Figura 2: Gráfico de sobrepeso durante I4 meses de avaliação

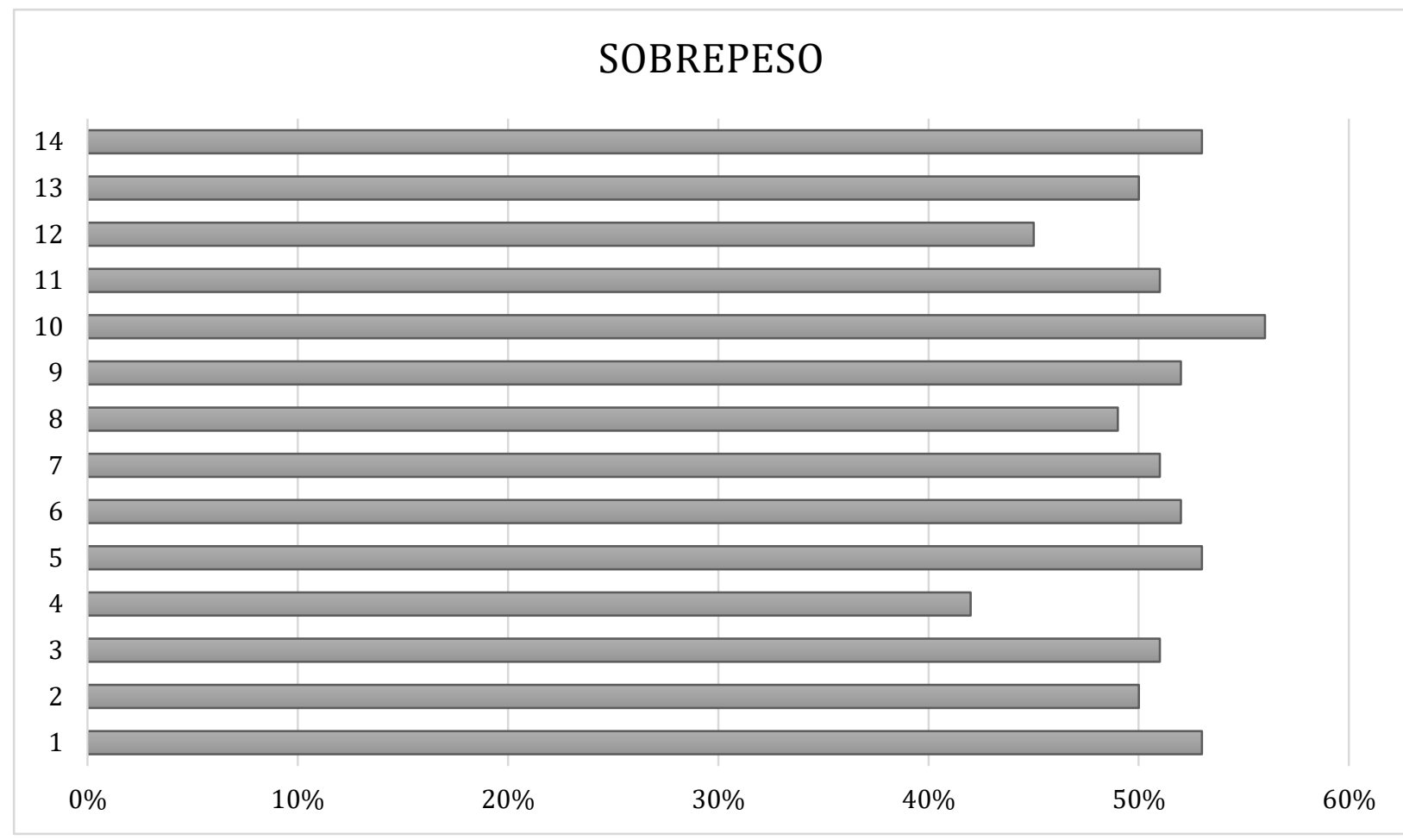

Quadro r: Índice de massa corporal em mulheres idosas do Nordeste do Brasil.

\begin{tabular}{|c|c|c|c|c|}
\hline \multicolumn{5}{|c|}{ IMC } \\
\hline \multirow{3}{*}{ MÊS/ANO } & BAIXO PESO & EUTROFIA & SOBREPESO & \multirow{3}{*}{ TOTAL } \\
\hline & QUANTIDAD & QUANTIDAD & QUANTIDAD & \\
\hline & $\mathrm{E}$ & $\mathrm{E}$ & $\mathrm{E}$ & \\
\hline JANEIRO/I9 & 66 & 113 & 200 & 379 \\
\hline FEVEREIRO/ & 6I & 148 & 2II & 420 \\
\hline 19 & & & & \\
\hline $\mathrm{MARÇO/19}$ & 60 & $\mathrm{I} 42$ & $2 \mathrm{II}$ & 413 \\
\hline ABRIL/I9 & 86 & I8I & 193 & 460 \\
\hline MAIO/19 & 59 & 168 & 253 & 480 \\
\hline JUNHO/19 & 45 & Io6 & 163 & 314 \\
\hline JULHO/19 & 87 & 233 & 325 & 645 \\
\hline AGOSTO/I9 & 93 & 204 & 291 & 588 \\
\hline
\end{tabular}




\begin{tabular}{|c|c|c|c|c|}
\hline $\begin{array}{c}\text { SETEMBRO/I } \\
9\end{array}$ & 84 & I84 & 289 & 557 \\
\hline OUTUBRO/I & 52 & I5I & 254 & 457 \\
9 & 44 & 122 & I77 & 343 \\
\hline NOVEMBRO & & & & 337 \\
\hline DEZEMBRO/ & 60 & 126 & I5I & 27 I \\
\hline I9 & 37 & 97 & I37 & 26 I \\
\hline
\end{tabular}

\section{DISCUSSÃO}

Os resultados evidenciam o perfil nutricional das idosas da Região Nordeste do Brasil, caracterizado pela alta prevalência de sobrepeso, seguida de eutrofia e baixo peso. Quando isolado, o sobrepeso oscila entre $40 \%$ e $60 \%$, sendo observado um período muito curto para um importante crescimento no ganho de peso, inclusive no mesmo ano.

Felicissimo $^{\text {12 }}$ ao tratar de conceitos bioquímicos diz que são percebidas alterações na composição orgânica dos indivíduos, em decorrência do aumento de gordura corporal, queda de massa muscular magra e alteração brusca no metabolismo hormonal (principalmente tireóide e crescimento), no caso das mulheres alterações provocadas através da menopausa.

Além disso, existem estudos que ressaltam que após 40 anos de idade, os indivíduos tendem perder massa óssea e assim reduzir sua estatura, principalmente por associar-se a sarcopenia que causa perda significativa da capacidade funcional. A prevalência de sarcopenia varia entre os 60 e 70 anos de idade, de 5 a $13 \%$, aumentando para $11 \%$ a $50 \%$ em pessoas com mais de $80 \operatorname{anos}^{13}$.

Dentre os diversos métodos usados para a avaliação nutricional destaca-se a antropometria. $\mathrm{O}$ índice de massa corporal (IMC), onde há combinação das medidas de peso e altura é o parâmetro mais usado para rastreamento de alterações do estado nutricional em todas as fases da vida, incluindo em pessoas idosas ${ }^{14}$. 
Existem diversas críticas ao pontos de corte de IMC, sendo a principal delas é que pontos de corte baseados em estudos e avaliação de indivíduos adultos e por isso não são adequados para classificação em idosos, devido às mudanças na composição corporal relacionadas ao envelhecimento ${ }^{15,16}$.

Em algumas situações, o indivíduo idoso não se mantem em pé, um dos requisitos para aferição de peso e estatura, sendo optado, assim, pelas circunferências do braço (CB), circunferência da panturrilha (CP) e circunferência abdominal (CA); Em seguida estimativas de peso e altura calculadas por meio de equações específicas ${ }^{17,18,19,20,21}$.

Considerando os pontos de corte para IMC em idosos, a Organização Pan Americana de Saúde (OPAS) ${ }^{22}$ define as seguintes referências: $<23 \mathrm{~kg} / \mathrm{m}^{2}$ baixo peso, 23 a $28 \mathrm{~kg} / \mathrm{m}^{2}$ peso normal, $28-30 \mathrm{~kg} / \mathrm{m}^{2}$ sobrepeso e $>30 \mathrm{~kg} / \mathrm{m}^{2}$ obesidade.

Enquanto, Lipchitz ${ }^{23}$ tem como classificações de IMC: $<22 \mathrm{~kg} / \mathrm{m}^{2}$ baixo peso, $22-27 \mathrm{~kg} / \mathrm{m}^{2}$ eutrofico e $>27 \mathrm{~kg} / \mathrm{m}^{2}$ sobrepeso, ou seja não há separação de sobrepeso e obesidade nessa avaliação. $\mathrm{A} \mathrm{OMS}^{24} \mathrm{faz}$ uso da mesma classificação do IMC que Lipchitz tornando assim esse ponto de corte o mais utilizado na prática clínica.

Existem limitações na avaliação do estado nutricional apenas pelo IMC, pois ele não permite a avaliação da composição corporal do idoso. Em casos de sobrepeso por exemplo não é possível afirmar com precisão se o indivíduo está com excesso de gordura corporal, uma vez que pode apresentar alto índice pela quantidade de massa muscular ${ }^{25}$.

Quando se fala em avaliação geriátrica ampla (AGA), a avaliação do estado nutricional é um dos itens principais, pois a identificação precoce de baixo peso, sobrepeso ou obesidade tem relação direta com comorbidades, morbimortalidade, funcionalidade e qualidade de vida. Observa-se também que a avaliação do idoso apresenta características particulares que as diferencia da avaliação nutricional dos demais grupos populacionais ${ }^{26}$.

A associação do IMC com outros parâmetros amplia a avaliação do estado nutricional do paciente. A circunferência da cintura e abdominal, por exemplo, tem forte correlação com o risco de doenças cardiovasculares, assim como a circunferência do pescoço. Enquanto a circunferência da panturrilha é considerada a medida mais sensível para avaliar e monitorar a perda de massa muscular em idosos, de ambos os sexos e atualmente tem pontos de corte revisados ${ }^{27,28}$. 


\section{CONCLUSÃO}

O presente estudo sugere que o estado nutricional de mulheres idosas residentes da Região Nordeste encontradas no SISVAN-WEB pode não evidenciar um resultado tão fidedigno do diagnostico nutricional, pois diversos parâmetros corroboram para melhor avaliação do idoso, não apenas o IMC e avaliação do consumo alimentar, que são os métodos que a plataforma utiliza.

O monitoramento do estado nutricional é essencial em todas as fases de vida e principalmente na fase idosa, pois eles tem mais probabilidade de adoecer. Portanto esse controle permite estabelecer estratégias para aumentar a qualidade de vida na assistência nutricional para essa população.

\section{REFERÊNCIAS}

1. BRASIL. Ministério da Saúde. Secretaria de Vigilância em Saúde. Departamento de Vigilância de Doenças e Agravos não Transmissíveis e Promoção da Saúde. Vigitel Brasil 2017: vigilância de fatores de risco e proteção para doenças crônicas por inquérito telefônico: estimativas sobre frequência e distribuição sociodemográfica de fatores de risco e proteção para doenças crônicas nas capitais dos 26 estados brasileiros e no Distrito Federal em 2017. Brasília: Ministério da Saúde, 2018.

2. Disponível em: https://censo202o.ibge.gov.br/2012-agencia-de-noticias/noticias/24036idosos-indicam-caminhos-para-uma-melhor-idade.html <Acesso em o6/II/2020 $>$.

3. Disponível em: https://agenciadenoticias.ibge.gov.br/agencia-noticias/2012-agencia-denoticias/noticias/2098onumero-de-idosos-cresce-18-em-5-anos-e-ultrapassa-30-milhoes-em2017\#: :text=As\%2omulheres\%20s\%C3\%A30\%20maioria\%2oexpressiva,(44\%25\%2odo\%2ogrupo) $\langle$ Acesso em 06/II/2020 $\rangle$.

4. AGUIAR, T, D, C et al. Avaliação nutricional e prevalência de doenças crônicas em população idosa, 2019.

5. AlVARENGA, M.; FIGUEIREDO, M.; TIMERMAN, F.; ANTONACCIO, C. Nutrição Comportamental. 2. ed. Barueri, SP: Manole, 2019. 
6. PALMA, Shelly Westphalen et al. Comparação do estado nutricional de idosos utilizando dois pontos de corte do índice de massa corporal. Saúde (Santa Maria), v. 42, n. I, p. I47-I54, 2016.

7. PEREIRA, Daniella Christiane Leite; LIMA, Sônia Maria Rolim Rosa. Prevalência de sobrepeso e obesidade em mulheres após a menopausa/Prevalence of overweight and obesity in postmenopausal women. Arquivos Médicos dos Hospitais e da Faculdade de Ciências Médicas da Santa Casa de São Paulo, v. 6o, n. I, p. I-6, 2018

8. BALD, Elisabete; ADAMI, Fernanda Scherer. AVALIAÇÃO NUTRICIONAL E PERFIL SÓCIO DEMOGRÁFICO DE IDOSOS INSTITUCIONALIZADOS. Revista Destaques Acadêmicos, v. II, n. 3, 2019.

9. MIRANDA, Rozinéia de Nazaré Alberto; DE PAIVA, Marinara Borges. Antropometria e consumo alimentar: identificador do estado nutricional de idosos. Nutrição Brasil, v. i8, n. 3, p. I4I-I5O, 2020.

Io. BANDEIRA, Geórgia Ferreira da Silva. Influência de determinantes sociais no estado nutricional de idosos assistidos na atenção básica em uma comunidade do Recife. 2017.

II. PEREIRA, Roberto Lucas Moura Ruben; SAMPAIO, Jéssica Pinheiro Mendes. Estado nutricional e práticas alimentares de idosos do Piauí: dados do Sistema de Vigilância Alimentar e Nutricional-SISVAN Web. Revista Eletrônica de Comunicação, Informação e Inovação em Saúde, v. 13, n. 4, 2019.

12. FELICISSIMO, Paulo; BRANCO, Jaime. Envelhecimento, metabolismo e nutrição no doente ortopédico. Revista Portuguesa de Ortopedia e Traumatologia, v. 25, n. 3, p. 186-192, 2017. 13. BALTHAR, Flaviane Cristina Farias. A relação do estado nutricional com o comportamento sedentário na população idosa portuguesa não institucionalizada como fator de risco a sarcopenia. 2020. Tese de Doutorado.

I4. LIMA, Marcos Felipe Silva de. Estado nutricional antropométrico, desenvolvimento e validação de equações para a estimativa de peso e estatura em idosos institucionalizados. 2019.

15. CARLESSO, Liziane Cássia. Consumo de gorduras saturadas e insaturadas por idosos diabéticos de Coronel Freitas/SC. Anuário Pesquisa e Extensão Unoesc Videira, v. 3, p. e19222el9222, 2018.

I6. SILVA, Geisane Sacramento. Avaliação do estado nutricional e do cardápio servido a idosos em uma instituição de longa permanência. 2018. 
17. CARDOZO, Natalia Rodrigues et al. Estado nutricional de idosos atendidos por unidades de saúde da família na cidade de Pelotas-RS. BRASPEN J, v. 32, n. I, p. 94-8, 2017.

18. COSTA, Fernanda Nascimento. Comparação do estado nutricional, qualidade de vida e capacidade funcional entre idosos institucionalizados e não institucionalizados. 2017.

19. BOCHI, Aline; ADAMI, Fernanda Scherer. Relação da qualidade de vida com o estado nutricional e faixa etária de idosos. Revista Brasileira de Ciências do Envelhecimento Humano, v. I4, n. I, 2017.

2o. SANT'ANNA, Bruna Calixto et al. Análise de concordância entre métodos para estimativa da estatura de idosos atendidos em ambulatório de nutrição. Revista Brasileira de Geriatria e Gerontologia, v. 21, n. 6, p. 718-723, 2018.

2I. Vitolo MR. Nutrição da gestação ao envelhecimento. 2 ed. Rio de Janeiro; Rubio; 2015.

22. OPAS. Organização Pan-Americana. XXXVI Reunión del Comitê Asesor de Ivestigaciones en Salud - Encuestra Multicêntrica - Salud Beinestar y Envejecimeiento (SABE) en América Latina e el Caribe - Informe preliminar. Disponível em: <URL:http://www.opas.org/program/sabe.htm.> 2002.

23. Lipschitz DA. Screening for nutritional status in the elderly. Prim Care. 21:55-67; 1994.

24. DE MAIO NASCIMENTO, Marcelo et al. Comparação e concordância de critérios à classificação do IMC de idosas fisicamente ativas, residentes no Sertão Nordestino. J Hum Growth Dev, v. 27, n. 3, p. 342-349, 2017.

25. Normas Técnicas da Vigilância Alimentar e Nutricional - SISVAN,2004.

26. ORTEGA, Natália Conessa et al. Associação entre os problemas da deglutição com as medidas de força muscular e velocidade de marcha em idosos octogenários: estudo de seguimento (Estudo FIBRA 2008/2009 e 2016/2017). 2020.

27. DA SILVA, Jacinayra Melo; DIAS, Savia Francisa Lopes. Análise da Capacidade Funcional e Estado Nutricional de Idosos Residentes em Asilo. Revista Ciência \& Saberes-Facema, v. 3, n. 4, p. 719-726, 2018.

28. DA CRUZ SILVA, Antonia Ilka; LANDIM, Liejy Agnes dos Santos Raposo. Perfil nutricional e estado de saúde de idosos fisicamente ativos. Nutrição Brasil, v. I9, n. I, p. 32-39, 2020 . 